\title{
4. ON ROTATING RING ORBITS IN GALAXIES
}

\author{
BERTIL LINDBLAD AND PER OLOF LINDBLAD \\ Stockholm Observatory, Saltsjöbaden, Sweden
}

If $\omega$ is the angular velocity of circular motions in a stellar system depending on the distance $R$ from the centre, and $\kappa$ is the frequency of oscillation in the radius vector for an orbit in the equatorial plane which differs slightly from a circular orbit, we may show [1] that in a coordinate system of angular speed

$$
\omega-\frac{1}{2} \kappa
$$

the orbit in question is a closed oval, in the first approximation an ellipse, with two opposite vertices and with its centre in the centre of the system. The conditions are illustrated in Fig. I, when $A A$ is the apsidal line of an orbit. The expression for $\kappa$ is

$$
\kappa=2 \sqrt{\omega(\omega-\bar{A})},
$$

where $A$ is Oort's constant of differential rotation. If the distribution of matter in the stellar system may be approximated by a sum of concentric spheroids with one and the same plane of symmetry, the density increasing with decreasing dimensions of the spheroids, we should expect $\kappa=2 \omega$ close to the centre, whereas in the outermost regions we must expect that $\kappa$ approaches to $\omega$. It is therefore likely that $\omega-\frac{1}{2} \kappa$ does not change appreciably within the system. Fig. 2 gives the run of $\kappa, \omega$, and $\omega-\frac{1}{2} \kappa$ for the galactic system according to the values of $\omega$ given by Kwee, Muller, and Westerhout [2]. In this case $\omega-\frac{1}{2} \kappa$ changes very slowly with $R$. A relative orbit has a quite important property, if $\omega-\frac{1}{2} \kappa$ is constant in the interval of $R$ covered by the orbit. In this case, if the centre of gravity of a cloud of free particles follows the orbit, and if the velocity dispersion is small, the particles will pursue orbits which differ only slightly from each other and from the central orbit, but on the other hand there will in general be considerable differential motions along the central orbit. This orbit is therefore the curve in space along which a cloud of free particles tends to disperse. The process is similar to the dispersion of the meteoric particles of a comet along its orbit.

If we follow a piece of matter in its motion along a slightly oblong orbit of this kind, the amount of matter per unit of the central angle $\theta$ will vary 


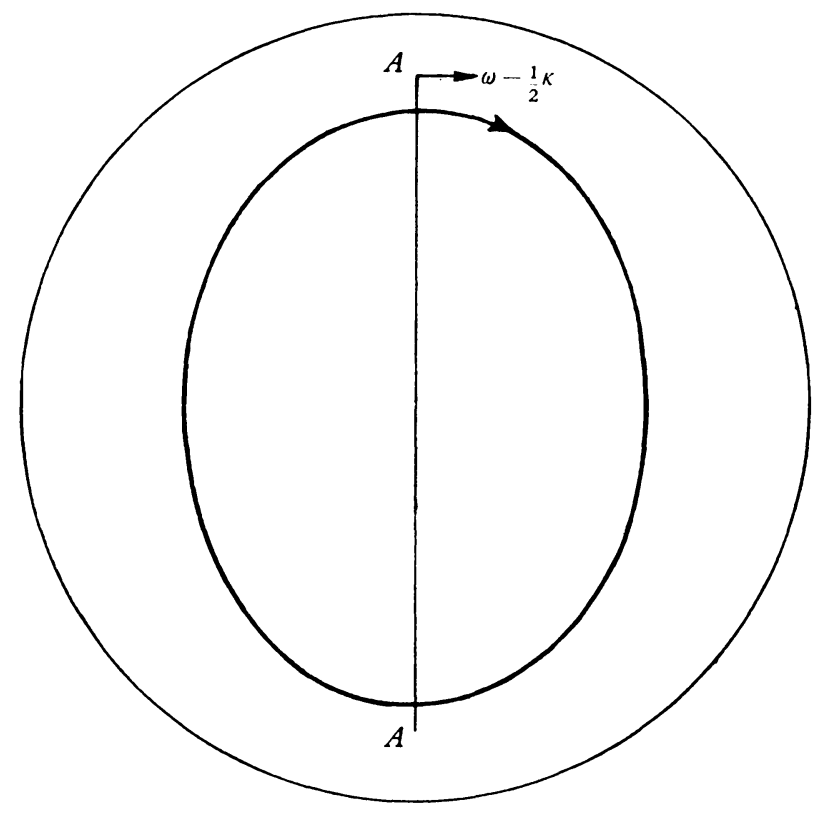

Fig. I.

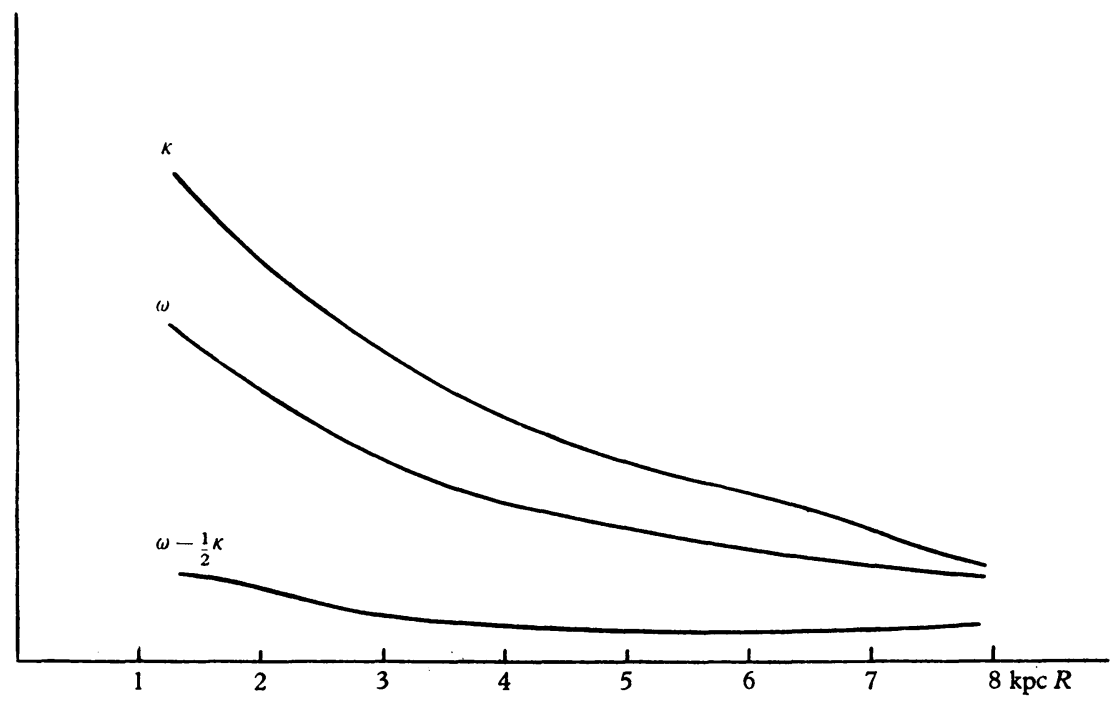

Fig. 2. 
due to the law of areas in the motion. In the case of a perfect mixing of matter along the orbit, which is of course an ideal case, but which will be approached as time increases, there will be steady maxima of density at the vertices (major axis) and steady minima at the ends of the minor axis. Due to this circumstance there will be secular disturbances of the motions in the ring, as well as on particles outside of the ring. In order to test this effect we have made computations on the motions of ring formations in a typical stellar system.

We assume as an interpolation formula in the interval of $R$ considered a central force of the form

$$
\frac{\partial \phi}{\partial R}=-\frac{a R}{\mathrm{I}+b R^{3}} \text {. }
$$

It is easily found that in this case $\omega-\frac{1}{2} \kappa$ is nearly constant in a region about $R=R_{0}$, where

$$
b R_{0}^{3}=2 \cdot 456 \text {. }
$$

If we choose $R_{0}=7 \mathrm{kpc}$, we have

$$
b=0.00716 \text {. }
$$

The value $\omega_{0}$ for $R=R_{0}$ may be chosen in accordance with the value derived by Kwee, Muller and Westerhout for $R=7 \mathrm{kpc}$ in the galactic system, $\omega_{0}=0 \cdot 0323 \mathrm{~km} / \mathrm{sec} \mathrm{pc}$, which gives

$$
a=0 \cdot 003606 \text {. }
$$

If we take as the unit of time $10^{6}$ years, as the unit of distance one kiloparsec, and express $\omega$ in $\mathrm{kpc} / \mathrm{Io}^{6} \mathrm{yrs} \mathrm{kpc}$, we find

$$
\frac{\partial \phi}{\partial R}=-\frac{0 \cdot 003768 R}{\mathrm{I}+0 \cdot 007 \mathrm{1} 6 R^{3}} \text {. }
$$

The total mass of the stellar system is found to be of the order $100.10^{9}$ solar masses.

At $R_{0}=7 \mathrm{kpc}$ we have

$$
\omega-\frac{1}{2} \kappa=0 \cdot 010454 \mathrm{kpc} / \mathrm{IO}^{6} \mathrm{yrs} \mathrm{kpc} \text {. }
$$

The orbit of a particle in the central field (4) in a co-ordinate system $\xi, \eta$ rotating with the angular speed (5) has been computed starting with the initial values

$$
\xi=0, \eta=+7 \cdot 7, \xi=+0 \cdot 12285, \dot{\eta}=0 .
$$

The resulting orbit is very nearly closed. The positions and velocities in the first quadrant ( $\xi$ and $\eta$ positive) were taken to define the orbital motion at intervals of time corresponding to $1 / 32$ of a period. The values in the other quadrants were formed by symmetry. In this way we find the initial distribution of thirty-two points along the periphery of the ring as shown in Fig. 3. 
Denoting with $x_{i}, y_{i}$ the co-ordinates of a point $i$ in a fixed co-ordinate system, the equations to be solved are as follows

$$
\left.\begin{array}{rl}
\frac{d^{2} x_{i}}{d t^{2}} & =-\frac{0.003768}{\mathrm{I}+0.007 \mathrm{I} 6 R_{i}^{3}} x_{i}-k \sum_{j \neq i} \frac{x_{i}-x_{j}}{\left[\left(x_{i}-x_{j}\right)^{2}+\left(y_{i}-y_{j}\right)^{2}\right]^{\frac{3}{2}}}, \\
\frac{d^{2} y_{i}}{d t^{2}} & =-\frac{0.003768}{\mathrm{I}+0 \cdot 007 \mathrm{I} 6 R_{i}^{3}} y_{i}-k \sum_{j \neq i} \frac{y_{i}-y_{j}}{\left[\left(x_{i}-x_{j}\right)^{2}+\left(y_{i}-y_{j}\right)^{2}\right]^{\frac{3}{2}}}, \\
R_{i} & =\left(x_{i}^{2}+y_{i}^{2}\right)^{\frac{1}{2}}, i, j=\mathrm{I}, 2, \ldots 3^{2 .}
\end{array}\right\}
$$

Choosing for each point a mass of $5 \cdot 10^{7}$ solar masses gives for the total mass of the ring $16.10^{8}$ solar masses, i.e. between $\mathrm{I}$ and $2 \%$ of the entire mass of the system. In this case we find

$$
k=2 \cdot 2462 \cdot 10^{-4} \text {. }
$$

In order to compensate the increase of central force on the particles due to the attraction of the ring itself, the initial velocities of the particles have been increased by a rotational motion which compensates the increase of central force for a circular arrangement of the thirty-two points with the radius $R=7 \mathrm{kpc}$.

The numerical integration of equations (6) was done with the Swedish electronic computer BESK, constructed and run by the Swedish Board of Calculating Machines at Stockholm. We are very much obliged to the Board for its kindness to allow us to use the machine for the present purpose. For working purposes the equations (6) were first transformed. On account of the symmetry in distribution and velocities of the particles the equations for the first sixteen may be written:

$$
\begin{aligned}
& \frac{d x_{i}}{d t}=\dot{x}_{i}, \\
& \frac{d y_{i}}{d t}=\dot{y}_{i}, \\
& \frac{d \dot{x}_{i}}{d t}=-\frac{a x_{i}}{f+b\left(x_{i}^{2}+y_{i}^{2}\right)^{\frac{3}{2}}}-\frac{c x_{i}}{4\left(x_{i}^{2}+y_{i}^{2}\right)^{\frac{3}{2}}}-\sum_{j \neq i} \frac{c\left(x_{i}-x_{j}\right)}{\left[\left(x_{i}-x_{j}\right)^{2}+\left(y_{i}-y_{j}\right)^{2}\right]^{\frac{3}{2}}} \\
& -\sum_{j \neq i} \frac{c\left(x_{i}+x_{j}\right)}{\left[\left(x_{i}+x_{j}\right)^{2}+\left(y_{i}+y_{j}\right)^{2}\right]^{\frac{3}{2}}} \cdot \\
& \frac{d \dot{y}_{i}}{d t}=-\frac{a y_{i}}{f+b\left(x_{i}^{2}+y_{i}^{2}\right)^{\frac{3}{2}}}-\frac{c y_{i}}{4\left(x_{i}^{2}+y_{i}^{2}\right)^{\frac{3}{2}}}-\sum_{j \neq i} \frac{c\left(y_{i}-y_{j}\right)}{\left[\left(x_{i}-x_{j}\right)^{2}+\left(y_{i}-y_{j}\right)^{2}\right]^{\frac{3}{2}}} \\
& \begin{array}{r}
-\sum_{j \neq i}\left[\frac{c\left(y_{i}+y_{j}\right)}{\left.\left(x_{i}+x_{j}\right)^{2}+\left(y_{i}+y_{j}\right)^{2}\right]^{\frac{3}{2}}}\right. \\
i, j=\mathrm{I}, 2, \ldots \mathrm{I} 6 .
\end{array}
\end{aligned}
$$


Keeping the numerical values of the constants in (6), the units of distance and time have been chosen so that the constants $a, b, c$ and $f$ satisfy the requirements of the machine. The equations (7) were then integrated by the method of Runge and Kutta with a constant full-step of $8.10^{6}$ years.

The results obtained for the single ring of thirty-two points are shown in Figs. 3-7. The number in each figure is the time expressed in the unit

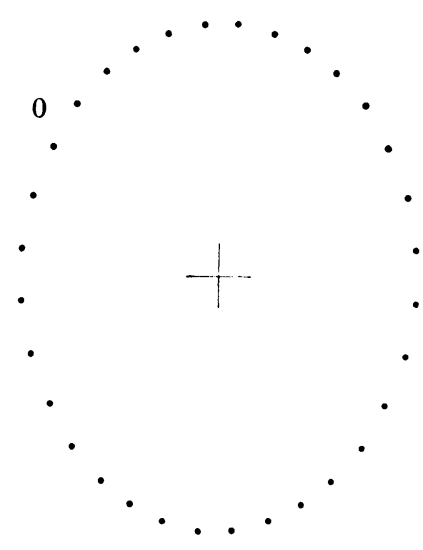

Fig. 3.

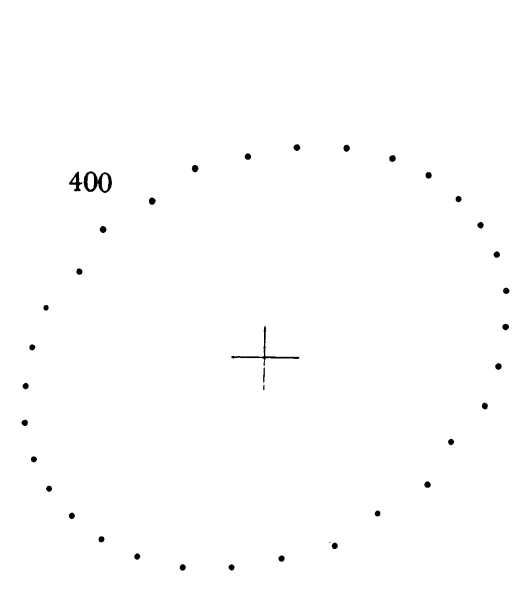

Fig. 5.

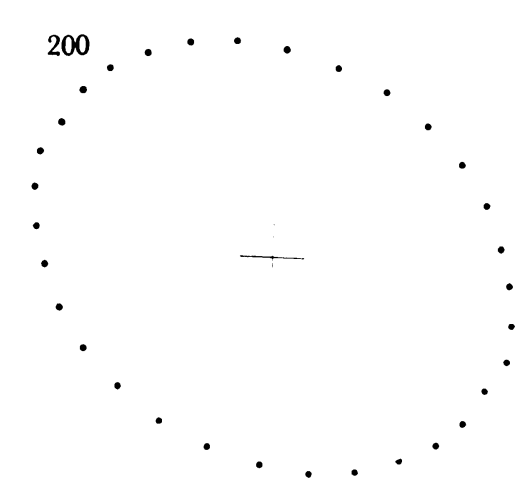

Fig. 4.

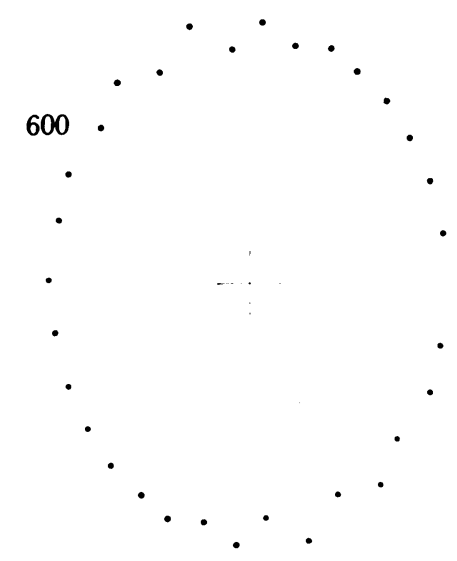

Fig. 6.

$10^{6}$ years. The disturbances are very small up to $t=400.10^{6}$ years. At $600.10^{6}$ years certain deviations from the smooth distribution occur and Fig. 7 shows that there is a tendency to break the ring into two separate ring-halves. At this time, however, certain points got so close together that 
the disturbing force exceeded the limits set by the machine with the present arrangements.

It may be remarked that this breaking up of the ring may be predicted analytically, as will be shown in a forthcoming paper on the present subject.

Of still greater interest is, however, the influence of the disturbing forces of a slightly oblong ring on neighbouring rings of matter, which may be assumed to have circular symmetry. In order to investigate this case we have distributed the thirty-two points on two rings, sixteen points on each. The outer ring is identical in form with the earlier one; the radius of the inner circular ring is $5.3 \mathrm{kpc}$. As before, the initial rotational velocities were adjusted to compensate the central force produced by the

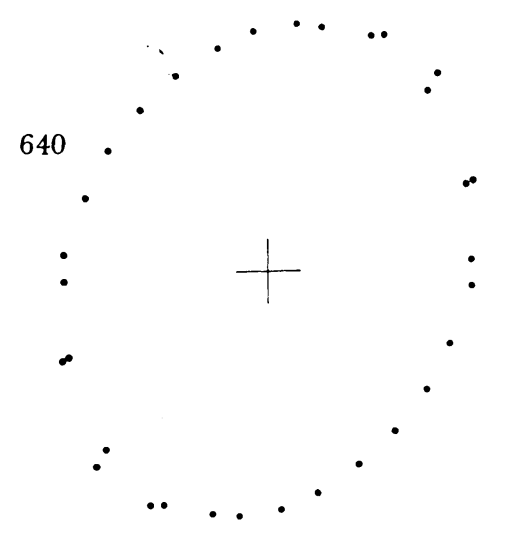

Fig. 7 .

combined attraction of the rings. The results of the computations are illustrated in Figs. 8-I I. In this case too the perturbations are small until a time of about $400.10^{6}$ years. At this time a widening and a beginning break-up of the inner ring is indicated. At $624 \cdot 10^{6}$ years this process is well marked. The inner ring has a tendency to break up into two halves, which, moreover, tend to become mixed with the matter of the outer ring. For larger values of $t$, coincidences between points set a limit to the calculation with the present arrangements.

Similar computations under varied initial conditions will be continued in the future. In particular it will be tried to cover larger intervals of time in the computations.

Already the preliminary results obtained here show beyond doubt the importance of such calculations for the problem of the development of 
spiral structure in galaxies. The theoretical concepts underlying an attempt to explain the spiral structure along these lines may be very briefly summarized as follows:

(I) The properties of symmetry which the spiral structure displays, and the general regularity of its pattern in other respects, for instance in the barred spirals, in spite of individual features which differ from one object

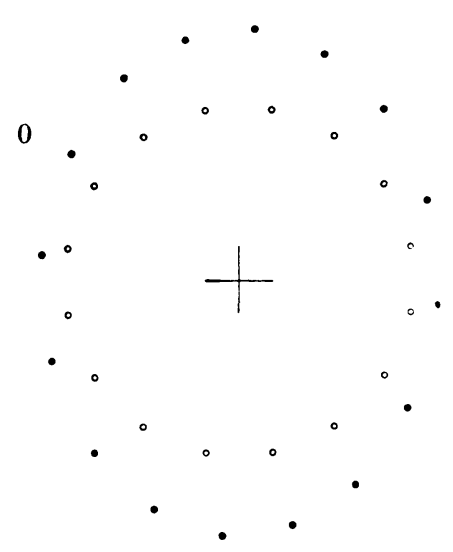

Fig. 8.

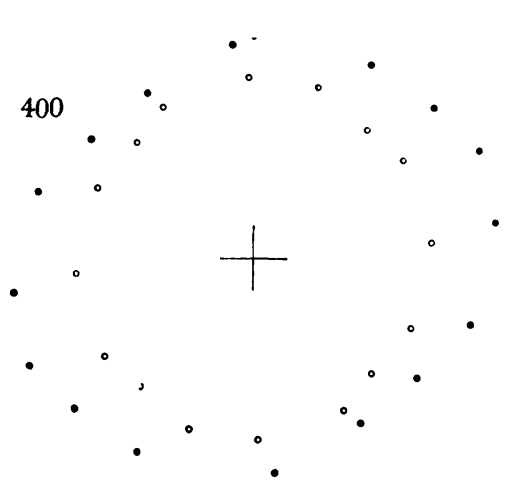

Fig. 10.

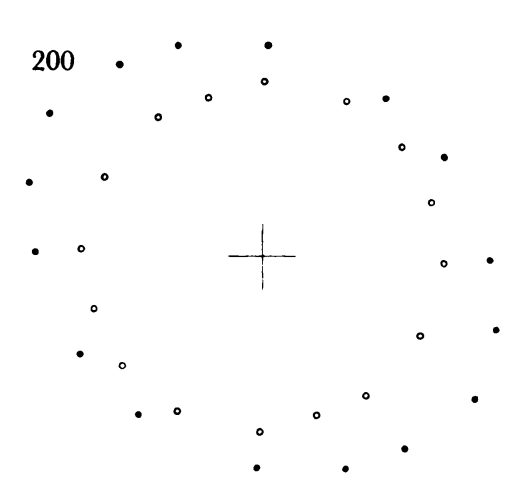

Fig. 9.

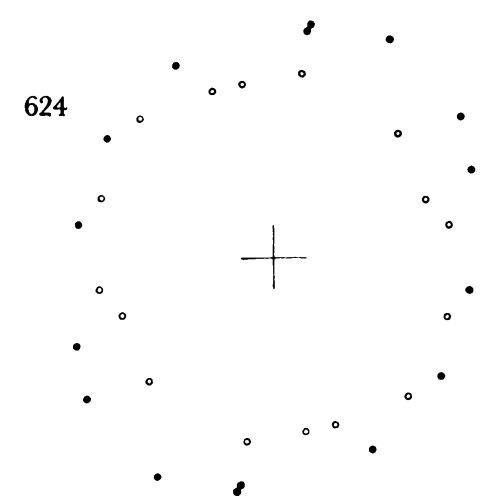

Fig. II.

to another, appear to indicate that the main cause of the spiral phenomenon should be sought for in gravitational disturbances. Other forces may not be able to produce effects of such regularity over the entire mass of the system.

(2) The process of formation of slightly elongated rings of matter is likely to be an entirely normal process in the evolution of a system. It 


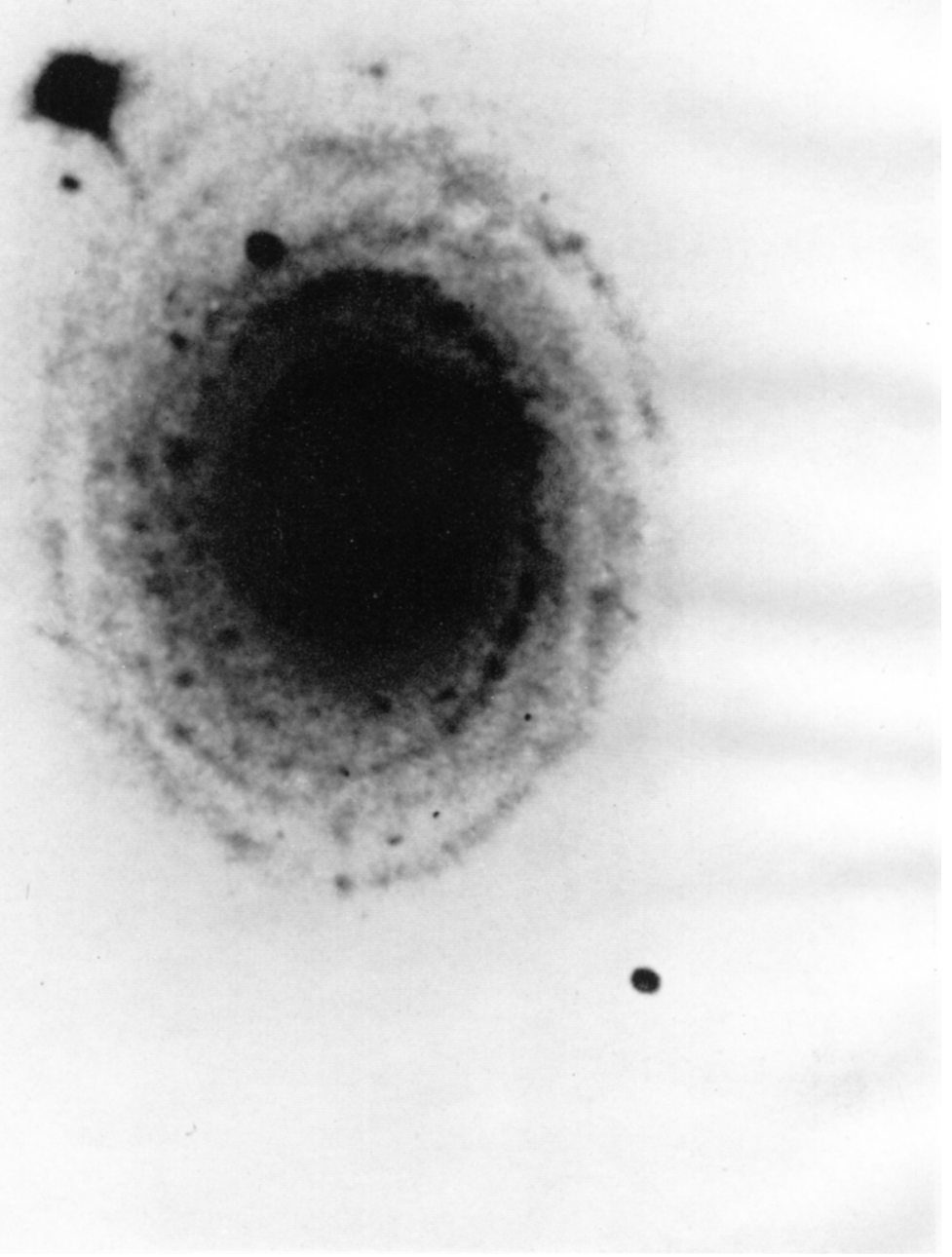

Fig. 12. The spiral galaxy NGC 488 . 
admits of an age of the spiral structure practically equal to that of the system itself. The development of spiral structure is in principle a slow process and is due to secular disturbances of the motion, which follow immediately from the average distribution of matter in the rings. This does not exclude that certain phases in the development of the structure may proceed rather rapidly.

(3) The symmetry of the spiral pattern follows immediately from the symmetry of the rings, and needs no auxiliary hypothesis.

A type of spiral system for which a direct application seems to be possible may be represented by the nebula $\mathrm{NGC}_{4} 88$ (Fig. 12). In this case we can readily imagine that the spiral pattern has originally consisted of a system of rings, which have been broken up by a symmetrical disturbing field of force. The apparent elongation of the outer rings may be at least partly real, because the inner spiral structure, which is overexposed in the picture, appears to be more nearly circular.

\section{REFERENGES}

[1] Stockholm obs. Ann. 18, no. 6, 1955 .

[2] Kwee, K. K., Muller, C. A. and Westerhout, G. B.A.N. 12, 2 I , 1954. 